\title{
Posttraumatische Knieendoprothetik
}

\section{Indikation und Implantatoptionen}

\section{Zusammenfassung}

Ziel der therapeutischen Bemühungen sollte die Vermeidung einer posttraumatischen Gonarthrose durch Wiederherstellung der verletzten anatomischen Strukturen sein. Die Indikation zur Knieendoprothese ist kritisch zu stellen, insbesondere vor dem 50. Lebensjahr. Alternative Behandlungsmethoden und Operationsverfahren sind abzuwägen. Die Implantation einer Knieendoprothese erfordert eine exakte Planung, präzise Operationstechnik und Balancierung der KapselBand-Strukturen und des Patellatrackings. Für den Behandlungserfolg mitentscheidend ist ein konsequent durchgeführter postoperativer Behandlungsplan mit effektiver Schmerztherapie.

\section{Schlüsselwörter}

Posttraumatische Gonarthrose · Indikation zur Knieendoprothese - Implantatoptionen . Ergebnisse nach Knie-TEP-Implantation ie Zahl der jährlich implantierten Kniegelenkendoprothesen nimmt kontinuierlich zu. Während in der eigenen Klinik 199042 Kniegelenke durch Endoprothesen versorgt wurden, waren es im Jahr 2000 bereits 374 (Zunahme um den Faktor 8,9). Im gleichen Zeitraum nahm die Zahl der Hüft-TEP-Implantationen von 211 auf $521 \mathrm{zu}$ (Zunahme um das 2,5Fache).

Im Jahr 1998 wurde die Zahl der in Deutschland eingesetzten Hüftendoprothesen auf 100.000 und die der Knieendoprothesen auf 40.000 geschätzt [8].

Nach einer 1997 in Deutschland durchgeführten Umfrage bei chirurgischen, unfallchirurgischen und orthopädischen Kliniken wurde die Indikation zur Knie-TEP in $73 \%$ der Fälle bei primären und in $27 \%$ bei sekundären Gonarthrosen (posttraumatische Gonarthrose $12 \%$, Arthrose bei rheumatoider Arthritis 15\%) gestellt [9]. Abhängig vom Schwerpunkt der Klinik variiert der Anteil der durch eine Endoprothese versorgten posttraumatischen Gonarthrose an der Gesamtzahl der Implantationen zwischen 3,5 [19] und 13,5\% (eigene Klinik).

Allen Veröffentlichungen zur Knieendoprothetik gemeinsam ist der nur geringe Anteil posttraumatischer Gonarthrosen. Die geringe Zahl der Fälle lässt keine differenzierte Betrachtung zu. So zählen langjährige Verläufe nach Meniskektomie, Kontusionen der Gelenkflächen und isolierte Kreuzbandverletzungen (Abb. 1) ebenso zu posttraumatischen Knieschäden wie komplexe Knietraumen mit ausgedehnten

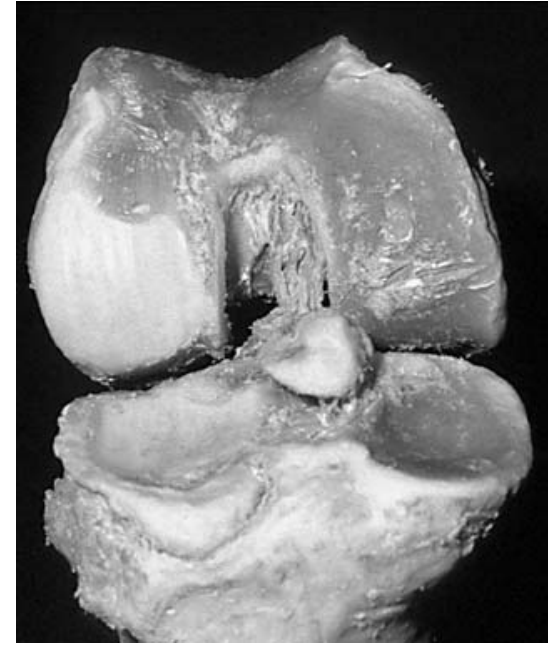

Abb. $1 \Delta$ Posttraumatische Gonarthrose nach knöchernem Ausriss des vorderen Kreuzbands

Weichteilschäden, bleibende muskuläre Defizite und ausgedehnte Narbenbildung. Die Erfolgsaussichten der Knieendoprothetik nach Meniskektomie und jahrzehntelangem Verlauf sind nicht mit einer schon nach wenigen Jahren notwendigen Knie-TEP-Implantation als Folge eines komplexen Knietraumas oder gar abgelaufenen Kniegelenkempyems nach offener Gelenkverletzung

\footnotetext{
๑) Springer-Verlag 2003

Prof.Dr. Christian Melzer Fachkrankenhaus für Orthopädie, Waldkrankenhaus Bad Düben, Gustav-Adolf-Straße 15A, 04849 Bad Düben, E-Mail:Info@dueben.mediclin.de, Tel.:034243-76600, Fax:034243-76603
} 


\section{Melzer $\cdot$ M.Gresens}

\section{Post-traumatic}

knee replacement surgery. Indications and implant options

\section{Abstract}

The objective of therapy efforts should be to avoid post-traumatic gonarthrosis by restoring the injured anatomical structures. The indications for implantation of a knee endoprothesis must be critically adhered to, especially in patients under 50 years of age. Alternative methods of treatment and surgery should be considered. Implantation of a knee endoprothesis requires accurate planning and high-precision surgery, and the capsular ligament structures must be balanced with patellar tracking. A factor that can contribute substantially to the success of therapy is a consistently followed postoperative therapy schedule providing for effective pain relief.

\section{Keywords}

Post-traumatic gonarthrosis · Indications for knee joint replacement - Implant options . Results · Total endoprosthesis implantation

\section{Posttraumatische Gonarthrose}

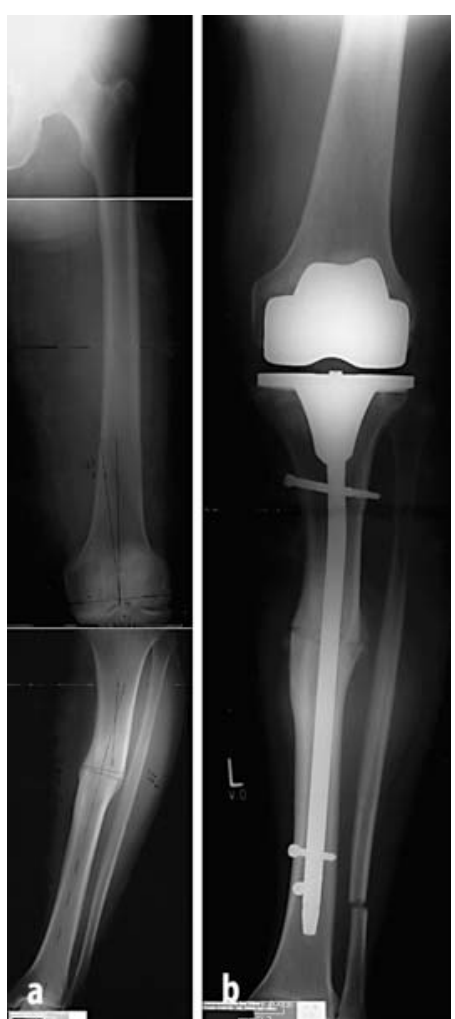

Abb. $2 \Delta$ Bikondyläre Knieendoprothese mit Unterschenkelkorrekturosteotomie und Stabilisierung durch einen Verrieglungsmarknagel

vergleichbar. Nur wenige Arbeiten befassen sich mit der Knieendoprothetik bei definierten posttraumatischen $\mathrm{Ge}$ lenkschäden [6].

\section{Alternativen zur Knieendoprothese}

Bevor die Indikation zur Knie-TEP gestellt wird, sind alternative Behandlungsmethoden in Betracht zu ziehen. Als nichtoperative Maßnahmen sind orthopädische Schuhzurichtungen (z. B. laterale Schuhranderhöhung bei Varusgonarthrose) und medikamentöse Therapieansätze (TNF- $\alpha$-Hemmer, Hyaluronsäure, Interleukin-1-Inhibition usw.) zu nennen. An operativen Alternativen zur Knieendoprothetik stehen arthroskopische Verfahren (Gelenklavage, Débridement, Abrasionsplastik, PridieBohrungen, Mikrofrakturierung, Karbonstifte u. a.), die Knorpel-(Knochen-)Transplantation (OATS und Mega-OATS, dorsaler Kondylentransfer, ACT/Tissue engineering, osteochondrale Allografts und Stammzellen- bzw. Gentherapie), Korrekturosteotomien bei Gelenk- und Achsenfehlstellungen, ggf. in Kombination mit der vorderen Kreuzbandplastik, die allogene Kniegelenktransplantation und die Arthrodese zur Verfügung [1, 2, $4,7,10,11,12,13,14,15,16,17]$.

\section{Präoperative Planung}

Bei der Indikation zur Knie-TEP-Implantation sind Begleit- und Vorerkrankungen zu berücksichtigen. Hierzu zählen Erkrankungen wie Diabetes melli-
Abb. 3 Anatomische Linien und Winkel für die Planung einer Knieendoprothese, aus Eulert $u$. Hassenpflug [6]
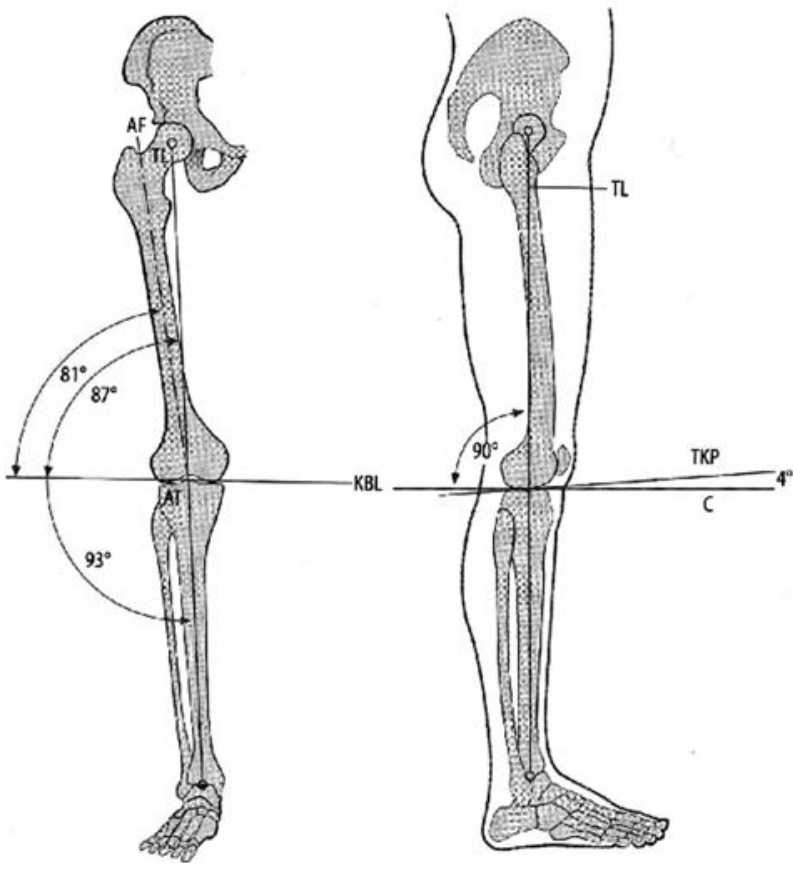


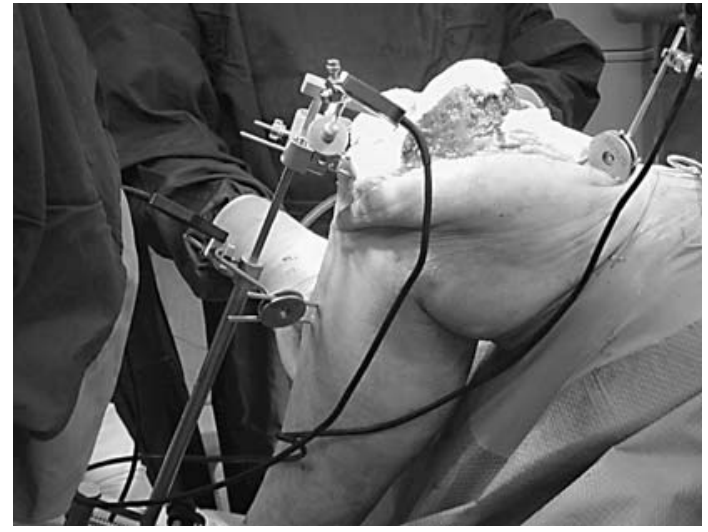

Abb. $4<$ Knie-TEP-Implantation mit der Anwendung eines Navigationssystems

tus, arterielle Verschlusskrankheit, Varikosis, Erkrankungen aus dem rheumatischen Formenkreis, Thrombosen und Embolien in der Vorgeschichte, Störungen der Blutgerinnung und neurologische Erkrankungen.

Bei der posttraumtischen Gonarthrose ist die Komplexität des Traumas (begleitende Gefäß- und/oder Nervenverletzungen, Kompartmentsyndrom, Weichteil- und/oder Gelenkinfekt, Lappenplastik und/oder Hauttransplantation, Narbenverlauf und aktuelle Durchblutungssituation) von Bedeutung. Aktuell eingenommene Medikamente (Kortison, ASS, Immunsuppressiva usw.) müssen bekannt sein. Auch das Körper-

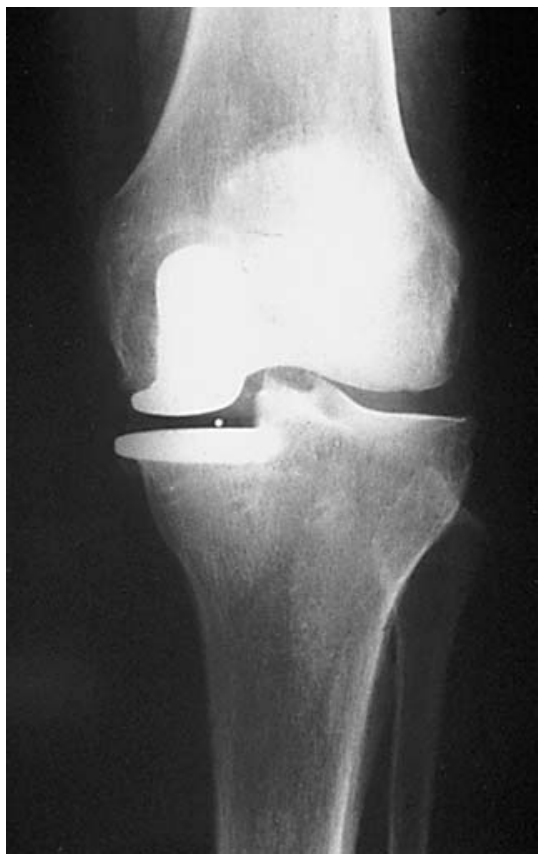

Abb. $5 \Delta$ Monokondyläre Knieendoprothese bei einer 64 -jährigen Patientin mit Varusgonarthrose gewicht und das Alter haben einen Einfluss auf das Operationsergebnis.

$\mathrm{Zu}$ den wichtigen klinischen Parametern zählen

- das Bewegungsausmaß,

- die Beinachsenstellung,

- die Stabilität,

- die muskuläre Funktion und

- das Patellofemoralgelenk (isoliertes patellofemorales Schmerzsyndrom, Hypo-, Hypermobilität, Lateralisation).

Neben der allgemeinen Laborroutinediagnostik sind v. a. Hinweise auf einen floriden Entzündungsprozess (BSG, CRP, Leukozytenzahl) zu berücksichtigen. In Zweifelsfällen sind ein 3-Phasenoder Antigranulozytenszintigramm unverzichtbar. Im Rahmen geltender Vorschriften besteht die Verpflichtung, auf die Möglichkeit der Eigenblut- und Plasmaspende hinzuweisen, sofern keine krankheitsbedingten oder allgemeinen Kontraindikationen bestehen.

Für die Planung unverzichtbar sind Röntgenaufnahmen im a.-p.- und seitlichen Strahlengang, eine Beinganzaufnahme im Stehen (evtl. 1-Bein-Stand) und Patella-Defilé-Aufnahmen in $30^{\circ}$, $60^{\circ}$ und $90^{\circ}$ Knieflexion. In bestimmten Fällen sind eine MRT- oder CT-Untersuchung erforderlich (unklare Infektsituation, Ausschluss eines Tumors, ausgedehnte Osteonekrosen, CT-basierte Navigation und/oder Robotic). Mit der Indikation zur Knieendoprothese müssen das Implantat und die Implantationstechnik festgelegt werden (mono- oder bikondylär, „fixed“ oder „mobile bearing“, mit oder ohne retropatellaren Gelenkflächenersatz, achsgeführtes Implantat; zementfrei oder zementiert oder hybrid).
Mit der Implantation verbundene zusätzliche operative Maßnahmen (Metallentfernung, Arthrolyse, ligamentäres oder Patellabalancing, Korrekturosteotomie; Abb. 2) müssen festgelegt werden, ebenso die Entscheidung, ob eine Blutsperre sinnvoll ist oder ein zusätzliches Risiko darstellt.

$\mathrm{Zu}$ fordern ist außerdem eine präoperativ angefertigte Planungsskizze mit Komponentenausrichtung und Bestimmung der Resektionsebenen (Mikulicz-Linie, anatomische Femur- und Tibiaachse, Kniebasislinie, „posterior slope“; Abb. 3), basierend auf einer intra- oder extramedullären Instrumentenausrichtung und Bestimmung der Implantatgrößen.

Die Wahl des Zugangs ist abhängig von

- vorbestehenden Narben,

- besonderen Weichteilbedingungen,

- Bewegungseinschränkungen und

- Deformitäten.

Neben der geraden anterioren Inzision kommen der mediale oder laterale parapatellare Zugang nach Payr, der Subvastuszugang, Rectus snip, Patella-turndown-Zugang und die Osteotomie der Tuberositas tibiae in Betracht $[8,20]$. Eine spezielle Planung erfordert auch der Einsatz eines Navigationssystems oder Operationsroboters (Abb. 4).

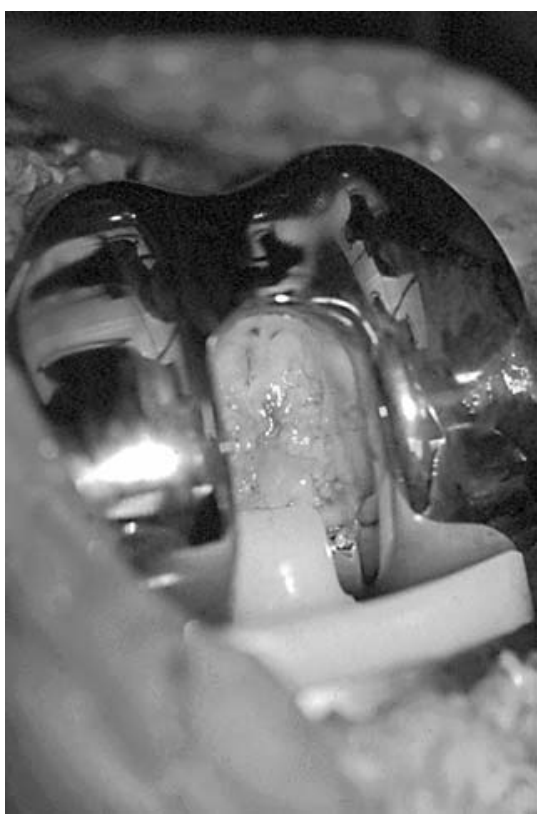

Abb. $6 \Delta$ Bikondyläre Knieendoprothese mit posterior stabilisierendem PE-Inlay 


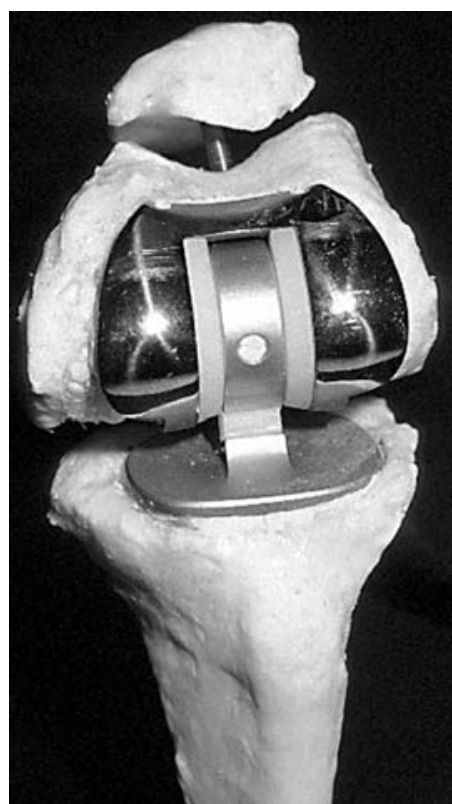

Abb. $7 \Delta$ Scharnierachsendoprothese

\section{Implantatoptionen}

Die Wahl des Gelenkimplantats ist abhängig vom

- Körpergewicht des Patienten,

- vorhandenen Kontrakturen,

- Gelenkstabilität

- knöchernen Destruktionen,

- Ausmaß der Achsenfehlstellung und Arthrose sowie

- Erfahrung des Operateurs.

Neben unikondylären (z. B. OxfordKnie; Abb. 5) stehen bikondyläre und achsgeführte Knieendoprothesen (Abb. 6) zur Verfügung.

Bikondyläre Endoprothesen können

- unter Erhalt des vorderen und hinteren Kreuzbands (z. B.LCS-Knie),

- mit Resektion des vorderen Kreuzbands (z. B. Foundation-Knie-TEP) oder

- mit Resektion beider Kreuzbänder (z. B.Endoprothese mit posterior stabilisierendem PE-Inlay)

implantiert werden.

Achsgeführte Knieendoprothesen stehen als

- $\quad$ Non-constrained- (z. B.GSB-Knieprothese),

- Semi-constrained- (Rotationsknieprothesen, z. B. Solution PLUS RT) und
- Constrained-Version (Scharnierachsprothesen, z. B. Blauth-Kniegelenk; Abb. 7)

zur Verfügung.

\section{Nachbehandlung}

Einen wesentlichen Einfluss auf die Ergebnisse nach Knieendoprothesenimplantation hat das postoperative Behandlungsregime. Durch eine effektive Schmerztherapie (Spinal-, Periduralka- theter, PCA-Pumpe) sind sowohl frühzeitige Bewegungsübungen als auch kontinuierliche passive Mobilisationsbehandlung (CPM) schon ab dem 1. postoperativen Tag möglich. Beim Auftreten eines Hämarthros sollte frühzeitig die Indikation zur Revision gestellt werden, nach unserer Erfahrung mit Eröffnung des Gelenks innerhalb der 1. postoperativen Woche und als arthroskopischer Eingriff ab der 2. postoperativen Woche. Bei einer Flexionsfähigkeit $<90^{\circ}$ sollte ab dem 5. postoperativen Tag die Indikation zur

Tabelle 1

Ergebnisse nach Foundation-Knie-TEP-Implantation bei primärer Gonarthrose, vorausgegangener Tibiakopfkorrekturosteotomie, posttraumatischer Gonarthrose und rheumatoider Arthritis

\begin{tabular}{lll} 
Diagnose & WOMAC & SF-36 \\
\hline Primäre Gonarthrose & 1,4 & $\begin{array}{l}72,1 \% \text { gut } \\
27,9 \% \text { mäßig }\end{array}$ \\
Nach Korrekturosteotomie & & $\begin{array}{l}75 \% \text { gut } \\
25 \% \text { mäßig }\end{array}$ \\
Posttraumatisch im Mittel 19 Jahre nach dem Trauma & 1,95 & $28,6 \%$ sehr gut \\
& & $42,9 \%$ gut \\
$28,5 \%$ mäßig \\
Rheumatoide Arthritis & 2 & $12,5 \%$ sehr gut \\
& & $75 \%$ gut \\
& & $12,5 \%$ mäßig
\end{tabular}

Tabelle 2

Kniegelenkbeweglichkeit in Abhängigkeit von vorbestehenden Befunden

\begin{tabular}{lllll}
$\begin{array}{l}\text { Bewegungs- } \\
\text { umfang [\%] }\end{array}$ & $\begin{array}{l}\text { Primäre } \\
\text { Gonarthrose } \\
{[\%]}\end{array}$ & $\begin{array}{l}\text { Nach Korrektur- } \\
\text { osteotomie [\%] }\end{array}$ & $\begin{array}{l}\text { Nach } \\
\text { posttraumatischer } \\
\text { Gonarthrose [\%] }\end{array}$ & $\begin{array}{l}\text { Rheumatoide } \\
\text { Arthritis [\%] }\end{array}$ \\
\hline$<90^{\circ}$ Flexion & 7 & 37,5 & 0 & 0 \\
$90-110^{\circ}$ Flexion & 53,5 & 25 & 42,9 & 62,5 \\
$>110^{\circ}$ Flexion & 39,5 & 37,5 & 57,1 & 37,5 \\
Streckdefizit $5^{\circ}$ & 4,6 & 4,6 & 0 & 0
\end{tabular}

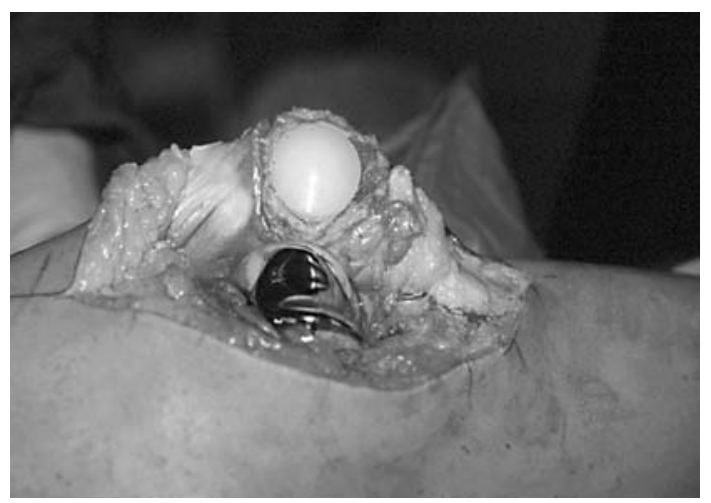

Abb. $8<$ Bikondyläre Knieendoprothese mit retropatellarem Gelenkflächenersatz 

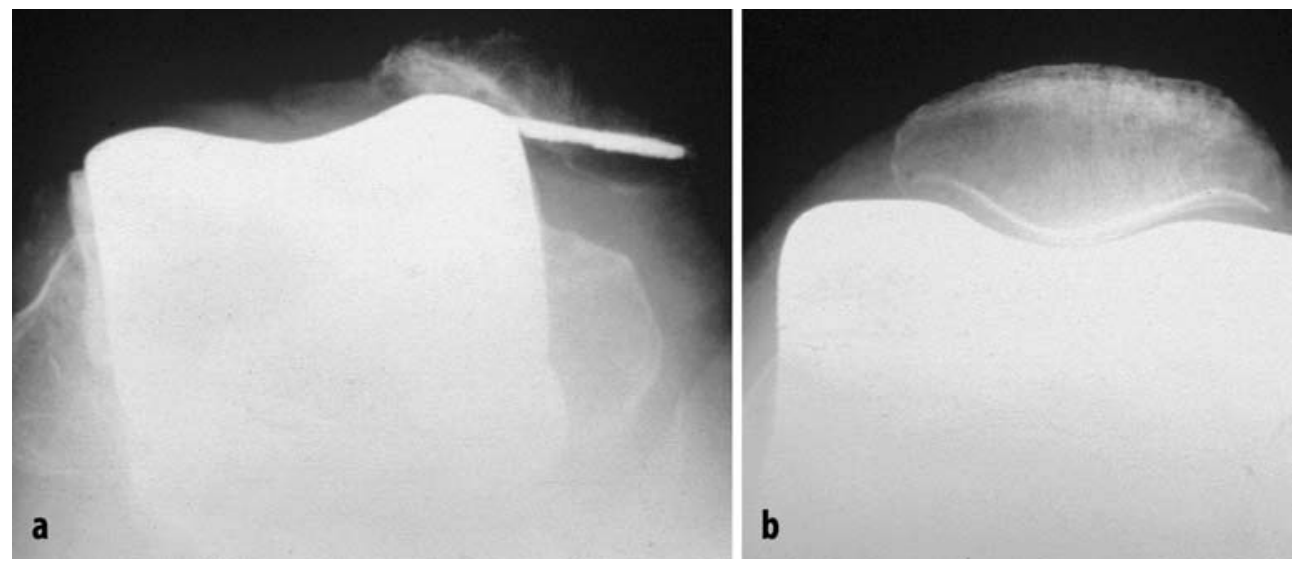

Abb. $9<$ Patellaluxation bei retropatellarem Gelenkflächenersatz und korrektes Alignement der Patella ohne Gelenkflächenersatz

Narkosemobilisation gestellt werden mit Platzierung eines Femoraliskatheters, um das erreichte Bewegungsausmaß langfristig sichern zu können. 12 Tage postoperativ sehen wir bei fehlender Kniestreckung die Indikation zur Anwendung einer Quengelschiene.

\section{Ergebnisse}

Die mit der Foundation-Knie-TEP (Fa. PLUS Endoprothetik) erreichten Ergebnisse mit einer Nachbeobachtungszeit von 2 Jahren und 4 Monaten sind in Tabelle 1 und 2 dargestellt. In Bezug auf die Beweglichkeit und den WOMAC-Score [18] sind bei posttraumatischen Gonarthrosen im Vergleich zur primären Gonarthrose bessere Resultate zu erzielen. Hervorzuheben ist v. a. in unserer Nachuntersuchungsserie das geringere Bewegungsausmaß nach vorangegangener Tibiakopfkorrekturosteotomie. Weniger deutlich sind die Unterschiede bei der Anwendung des MOS-SF-36 [3]. In weiteren Vergleichsstudien konnten wir keinen Unterschied mit und ohne gleichzeitigen retropatellaren Gelenkflächenersatz feststellen (Abb. 8). Entscheidend ist nicht der retropatellare Gelenkflächenersatz, sondern das $\mathrm{Pa}$ tellabalancing, ggf. durch zusätzliche operative Maßnahmen (Abb. 9).

\section{Komplikationen}

Es muss zwischen

- frühen Komplikationen und

- Spätkomplikationen

unterschieden werden. Bei den frühen Komplikationen steht das Auftreten einer Thrombose (TBVT) mit 14\% (Lungenem- bolie $2 \%$, fulminant $1 \%$ ) an erster Stelle, gefolgt von einem postoperativen Hämarthros mit $13 \%$ (2,5\% revisionsbedürftig), einer oberflächlichen Wundheilungsstörung mit 4\% und der Notwendigkeit der Narkosemobilisation mit 4\% [19].

$\mathrm{Zu}$ den Spätkomplikationen nach einer Beobachtungszeit von 6,2 Jahren zählen

- die aseptische Lockerung mit 8,1\%,

- Instabilität mit 4,8\%,

- $\quad$ tiefe Infektion mit 3,3\%,

- Abriss des Kniestreckapparats oder Patellalateralisation in 2,4\%,

- $\quad$ periprothetische Fraktur mit 0,9\% und

- Luxation mit 0,5\% [19].

Im eigenen Krankengut beträgt die Überlebensrate der Foundation-KnieTEP nach 2 Jahren und 4 Monaten 92,8\%.

\section{Literatur}

1. Bobic V (1999) Die Verwendung von autologen Knochen-Knorpel-Transplantaten in der Behandlung von Gelenkknorpelläsionen. Orthopäde 28: 19-25

2. Brittberg $M$, Lindahl $A$, Nilsson $A, O$ hlsson $G$, Isaksson 0, Peterson L (1994) Treatment of deep cartilage defects in the knee with autologous chonrocyte transplantation. N Engl J Med 331:889-895

3. Bullinger M, Kirchberger I (1998) SF-36-Fragebogen zum Gesundheitszustand. Hogrefe, Göttingen

4. Coventry MB (1993) Alternatives to total knee arthroplasty. In: Rand JA (ed) Total knee arthroplasty. Raven Press, New York, pp 67-84

5. Eulert J, Hassenpflug J (2000) Praxis der Endoprothetik. Springer, Berlin Heidelberg New York

6. Gerich T, Bosch U, Schmidt E, Lobenhoffer $P$, Krettek C (2001) Kniegelenksendoprothetik nach Tibiakopffrakturen. Mittelfristige Ergebnisse einer Kohortenanalyse.Unfallchirurg 104: 414-419
7. Imhoff AB, Burkart A, Öttl GM (1999) Der posteriore Femurkondylentransfer. Orthopäde 28 : 45-51

8. Jerosch J, Heisel J (1998) Knieendoprothetik. Springer, Berlin Heidelberg New York

9. Jerosch J, Fuchs S, Heisel J (1997) Knieendoprothetik - eine Standortsbestimmung. Dtsch Ärztebl 94:449-455

10. Johnson L (1986c) Arthroscopic abrasion arthroplasty, historical and pathologic perspective: present status arthroscopy. Arthroscopy 2:54-69

11. Jungmichel D, Weber H, Gatzsche L (1988) Gelenkwaschung - eine Behandlungsmöglichkeit bei aktivierter Arthrose. Beitr Orthop Traumatol 35:511-517

12. Lexer $E$ (1909) Über Gelenktransplantation. Langenbecks Arch Klin Chir 90:263

13. Messner K (1999) Knorpelersatzoperationen mit vorkultivierten Zellen. Orthopäde 28: 61-67

14. Möller HD, Evans CH (1999) Gentherapeutische Ansätze in der Arthrosebehandlung. Orthopäde 28:76-81

15. Pridie KH (1959) A method of resurfacing osteoarthritic knee joints.J Bone Joint Surg Br 41: 618-619

16. Schultz W, Gobel D (1999) Articular cartilage regeneration of the knee joint after proximal tibial valgus osteotomy: a prospective study of different intra- and extraarticular operative techniques. Knee Surg Sports Traumatol Arthrosc 7:29-36

17. Shariaree $H, O^{\prime}$ Conner RL, Nottage W (1982) Seven years follow up on arthroscopic debridement of the degenerative knee. Field View 1:1

18. Stucki G, Meier D, Stucki S, Michel BA, Tyndall AG, Dick W, Theiller R (1996) Evaluation einer deutschen Version des WOMAC (Western Ontario and McMaster Universities) Arthrose Index.Z Rheumatol 55:40-49

19. Stukenborg-Colsman C, Ostermeier S, Wirth CJ (2000) Knieendoprothetik - Biomechanische Voraussetzungen und Konsequenzen. Orthopäde 29:722-726

20. Wehrli U, Bosch U (2001) Lateraler Zugang für den endoprothetischen Kniegelenksersatz bei der so genannten Valgusarthrose. Operat Orthop Traumatol 13:54-69 